\title{
Sistema de Monitoramento Inteligente da COVID-19 em SP
}

\author{
Alessandro S. Santos* \\ Igor C. Teixeira, Leandro Avanço, Rodrigo Neves, Icaro Gonçales, Angelina Inacio ${ }^{\dagger}$ \\ Instituto de Pesquisas Tecnológicas do Estado de São Paulo - IPT \\ São Paulo, São Paulo
}

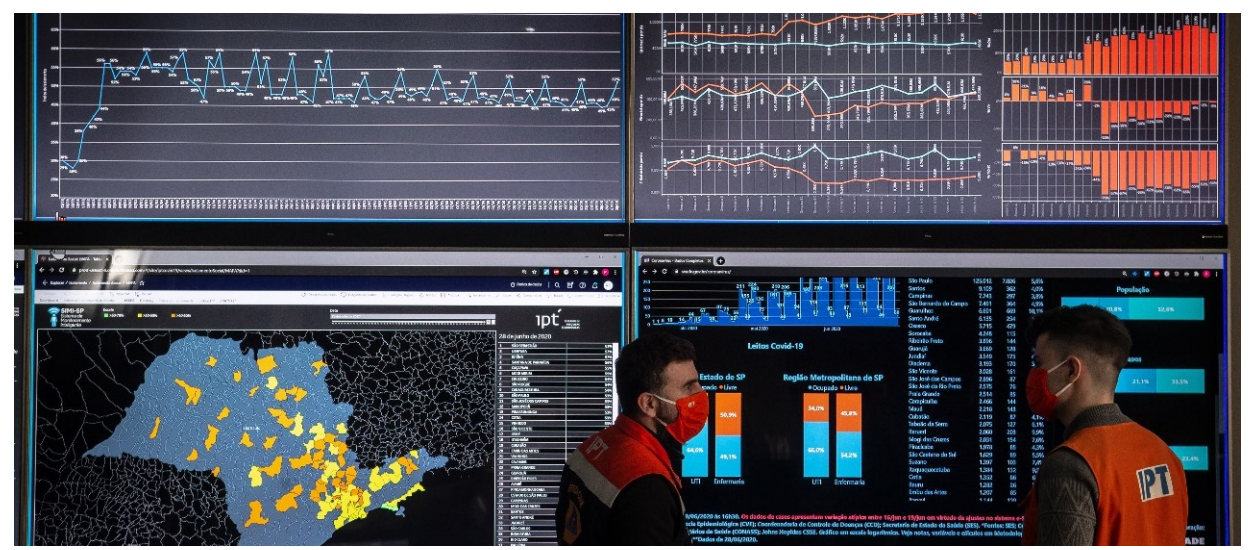

Figure 1: Sala de Situação com as visões da aplicação

\begin{abstract}
São Paulo was one of the first states affected by COVID-19 in Brazil. The Intelligent Monitoring System (SIMI) was an important milestone to face the pandemic, whose mission was to consolidate and integrate data on vehicular and people mobility, epidemiological situation, and economic status to support the state government in its strategic decisions. Overcoming the challenges of integration, anonymity, privacy, and multidisciplinarity were essential to provide results for analyzing scenarios and better information for decision-making. SIMI indicators impacted the entire São Paulo society, which defined criteria for flexibilization or restriction of economic sectors or supporting strategic changes to face the pandemic.
\end{abstract}

\section{KEYWORDS}

COVID-19, Business Intelligence, IoT, BigData, Data visualization

\section{INTRODUÇÃO}

A pandemia de COVID-19 revelou a necessidade de obter informações estratégicas para a tomada de decisão baseada em dados para o gerenciamento eficiente de pandemias.

\footnotetext{
*Coordenador do mestrado profissional em computação aplicada e pesquisador do IPT lattes.cnpq.br/9738704704763672

†Pesquisadores e mestrandos em Computação Aplicada do IPT Igor:lattes.cnpq.br/8760860893828089; Leandro:lattes.cnpq.br/4001364982287130 Ícaro:lattes.cnpq.br/0674169941950740; Angelina:lattes.cnpq.br/3919028230682858; Rodrigo:lattes.cnpq.br/1293226721509041
}

In: XX Workshop de Ferramentas e Aplicações (WFA 2021), Minas Gerais, Brasil. Anais Estendidos do Simpósio Brasileiro de Sistemas Multimídia e Web (WebMedia). Porto Alegre: Sociedade Brasileira de Computação, 2021.

(c) 2021 SBC - Sociedade Brasileira de Computação.

ISSN 2596-1683
O monitoramento da mobilidade populacional é um dos desafios para o enfrentamento da pandemia [5], em que diferentes tecnologias e cenários podem monitorar os movimentos populacionais para fornecer informações sobre os resultados das políticas públicas, promovendo maior resiliência para a cidade [2].

O Sistema de Informações e Monitoramento Inteligente (SIMI) é uma aplicação WEB que se destina a apoiar a formulação e avaliação das ações do Estado de São Paulo para enfrentamento da pandemia, limitando-se a dados anonimizados, dentro das premissas da Lei Geral de Proteção de Dados Pessoais, provendo informações na WEB para a sociedade e na sala de situação para uso do Comitê de Contingência (Fig. 1).

O SIMI teve como meta ser uma importante ferramenta de apoio à gestão da pandemia, fornecendo dados consistentes, integrados e com visões coerentes com as necessidades do governo, seja para avaliar o resultado de politicas relacionadas à mobilidade veicular, circulação de pessoas, impactos na economia e situação epidemiológica. A Fig. 2 apresenta a arquitetura geral da aplicação. Além disso, a aplicação teve apoio de agências de fomento ${ }^{1}$.

\section{ARQUITETURA E FUNCIONALIDADES}

O desenvolvimento da aplicação e a montagem da sala de situação se beneficiou do uso de métodos ágeis, empregando um ciclo interativo que envolveu: pesquisa de fontes de dados; validação de dados e entendimento do propósito da base; integração de camadas de

\footnotetext{
${ }^{1}$ Este trabalho foi suportado pelo Projeto FAPESP n ${ }^{\circ}$ 2017/50343-2, intitulado Plano de Desenvolvimento Institucional na área de Transformação Digital: Manufatura Avançada e Cidades Inteligentes e Sustentáveis (PDIP). Este também teve colaboração do INCT da Internet do Futuro para Cidades Inteligentes, financiado por CNPq (proc. 465446/2014-0), Coordenação de Aperfeiçoamento de Pessoal de Nível Superior - Brasil (CAPES) - Código de Financiamento 001 e FAPESP (procs. 14/50937-1 e 15/24485-9)
} 


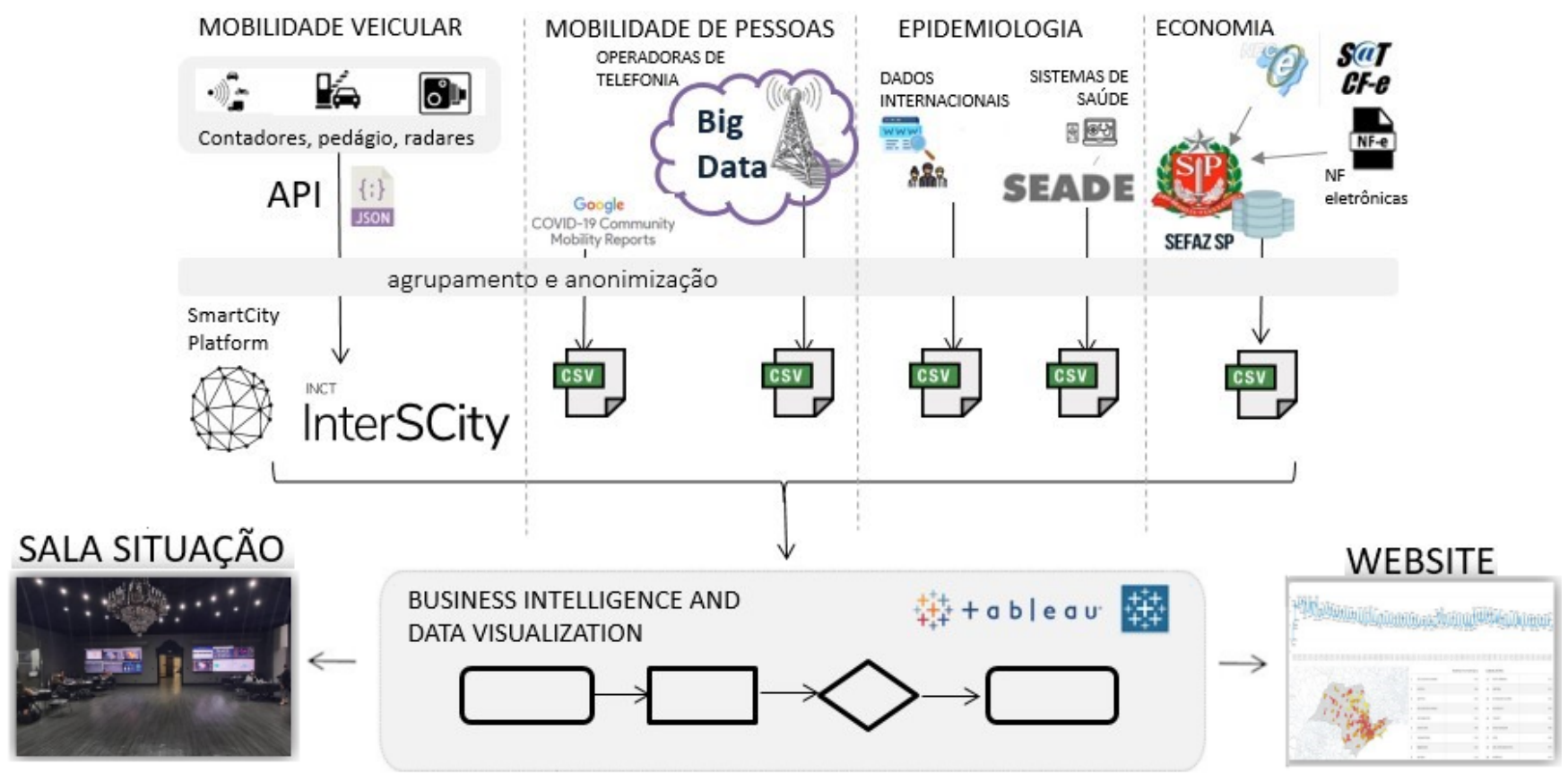

Figure 2: Arquitetura geral da aplicação

apresentação; coleta sistematizada de dados; visualização de dados com técnicas de Business Intelligence.

A infraestrutura principal é baseada em nuvem, contendo mecanismos de integração com dispositivos IoT e Plataforma de Cidade Inteligentes InterSCity [1]. A plataforma InterSCity é um projeto de código aberto licenciado sob MPL 2.0, desenvolvido e liderado pelo Instituto de Matemática Estatística da USP, que em conjunto com pesquisadores do IPT, customizaram a plataforma de forma a garantir redundância, alta disponibilidade e segurança.

Todos os cenários exigiram um ciclo de montagem de dashboards apropriados e focados na tomada de decisão. Destacamos quatro grandes cenários: (i) Isolamento social via big data das operadoras de telefonia; (ii) Mobilidade veicular intraestadual; (iii) Situação epidemiológica; (iv) Situação econômica. Em [8] podem ser vistos os vários desafios para sua implantação e mais detalhes da arquitetura. $\mathrm{O}$ vídeo youtu.be/5k88B6Ajtnw apresenta algumas visualizações das principais funcionalidades da aplicação.

\subsection{Isolamento social via Big Data}

A infraestrutura de telecomunicações tem sido a grande parceira tecnológica para capturar padrões de mobilidade em várias partes do mundo [6]. Este suporte permite monitorar a movimentação de pessoas por meio de infraestrutura celular, e tem sido usado e demonstrado bons resultados no subsídio à tomada de decisão.

O IPT estabeleceu um acordo de cooperação com operadoras de telefonia, integrando-se ao ambiente de Big Data das operadoras para obter dados sobre isolamento social. O índice de isolamento social é construído com informações provenientes das Estações de Rádio Base, que identificavam quando o celular migrava de antenas, sinalizando assim que o mesmo deixou o isolamento social.
As operadoras de rede móvel coletam dados de sua infraestrutura, integrando em seus ambientes de Big Data e aplica procedimentos de agrupamento por regiões com anonimização para envio diário das informações ao SIMI, que cria a visualização dos dados de forma estratégica para o comitê de contingência.

Ainda foram utilizadas informações do Google Mobility Report como forma de mapeamento da circulação das pessoas em segmentos setoriais, tais como varejo e lazer: transporte público, parques, farmácias e mercados, etc.

\subsection{Mobilidade veicular intraestadual}

Foi fundamental mapear os fluxos interurbanos de veículos para capturar os padrões de propagação do SARS-COV-2 no Estado e apoiar o processo de decisão.

A plataforma InterSCity foi implantada como receptora de dados de dispositivos de Internet das Coisas (IoT) já instalados em rodovias paulistas (contadores de tráfego, radares de velocidade, equipamentos em praças de pedágio). Sistemas e equipamentos de rodovias enviam dados anônimos para a plataforma, consolidando os dados de várias regiões do estado e integrando-os ao SIMI, de 3 em 3 horas. Esta consolidação foi agrupada em raios concêntricos, a partir da capital paulista, possibilitando assim a visualização do fluxo de veículos entre regiões, mapeando as regiões mais críticas para o contágio. (Fig. 3).

\subsection{Situação epidemiológica}

Os dados de saúde foram coletados por meio de integração com bases de dados nacionais e regionais. Toda esta integração foi realizada pela Fundação Sistema Estadual de Análise de Dados (SEADE), 


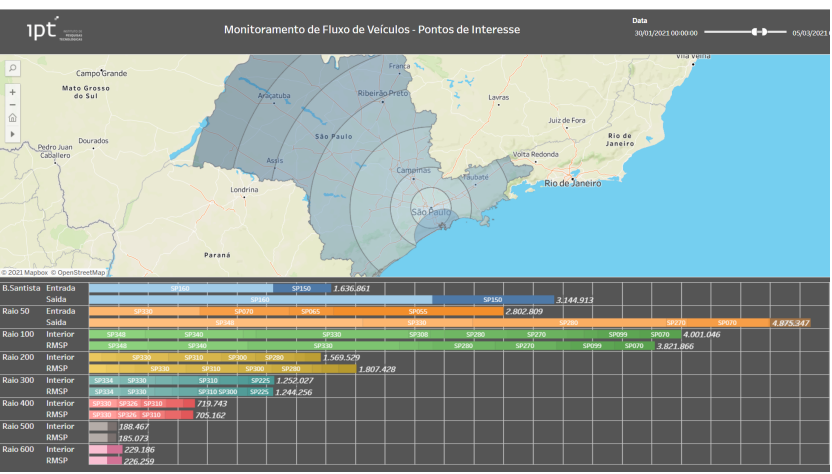

Figure 3: Mapa de fluxo de veículos agrupado por raios

que consolidou os dados e integrou ao SIMI. Além dos dados regionais, o SIMI contou com a integração com APIs de sites internacionais ou mesmo por coleta manual por meio de voluntários e colaboradores, o que permitiu criar dashboards tradicionais de casos e óbitos, distribuídos por cidades e em escala temporal, sexo, raça, idade, dentre outras perspectivas.

Além dos modelos tradicionais, foi possível realizar visualizações relacionando a mobilidade e a situação epidemiológica, associando a variação percentual da mobilidade em setores como varejo e lazer (provenientes do Google Mobility Report), com dados epidemiológicos, a fim de identificar o quanto as restrições impactaram no número de casos [9](Fig. 4).

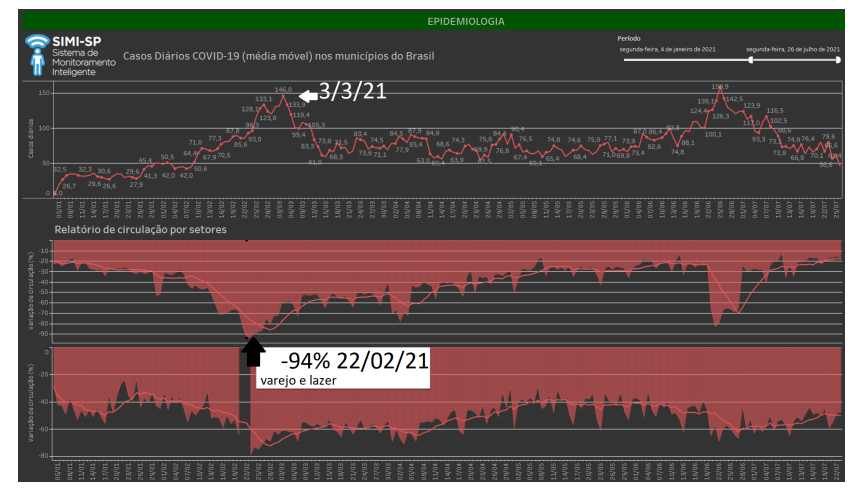

Figure 4: Mobilidade e Epidemiologia em Araraquara

\subsection{Situação econômica}

O monitoramento foi feito por meio das emissões de notas e cupons fiscais eletrônicos, instituídos por programas fiscais (NFC-e, Sat$\mathrm{CFe}, \mathrm{NFe}$ ), que são encaminhados diariamente para Secretarias de Fazenda (SEFAZ). Estes dados compõem um Data Warehouse com informações para monitorar a arrecadação fiscal, que são consolidados e agrupados por categorias e regiões de interesse, para gerar estimativas da atividade econômica, de forma a respeitar regulamentação que proteja a anonimização e sigilo fiscal. Estes dados são enviados semanalmente ao SIMI.

A Fig. 5 demonstra o Dashboard estruturado com a ferramenta Tableau, que cria visões da evolução e recuperação econômica com comparações entre fases pré e durante a pandemia, onde o mapa apresenta a situação semanal nas diferentes regiões (semana 12 - inicio da quarentena em 2020) e os gráficos apresentam a evolução temporal da variação percentual da quantidade e do valor das emissões de notas.

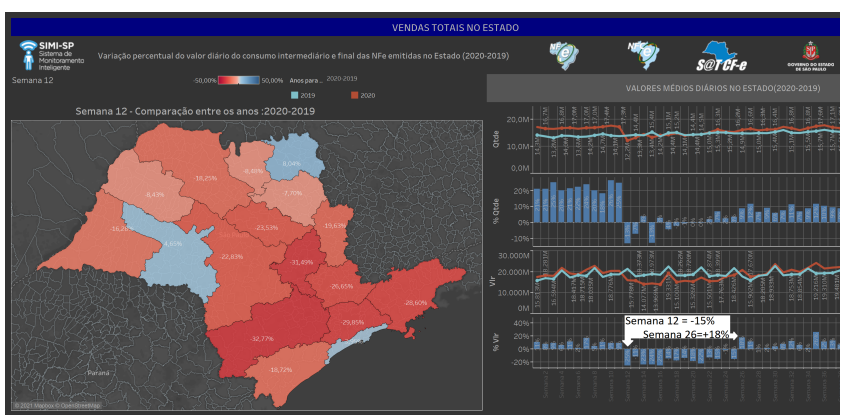

Figure 5: Visão econômica na $\mathbf{1}^{\mathrm{a}}$ semana de quarentena

\section{RESULTADOS E IMPACTOS}

Os dados providos pelo SIMI fazem parte da pauta de discussão do centro de contingência do coronavírus em São Paulo, subsidiando as ações e impactando diretamente o Plano São Paulo. Este plano determina cores regionais baseado em níveis de risco que sinalizam alteração na operação de setores econômicos, definidos com base no indicadores providos.

De forma geral, elencamos os seguintes resultados específicos:

- Com a abordagem de monitoramento epidemiológico, avaliouse a disseminação e a relação com medidas de controle da mobilidade e demais ações preventivas. Assim permite análises espaço temporais da disseminação do vírus, colaborando com o planejamento e alocação de recursos hospitalares e no apoio humanitário. A cidade de Araraquara, por exemplo, promoveu lockdown em dois momentos de 2021, evidenciados com percentuais de redução na mobilidade do setor de varejo e lazer, que chegaram a $-94 \%$, o que promoveu uma redução na média móvel de casos após 10 dias do inicio do lockdown (Fig. 4).

- Com abordagem do monitoramento da mobilidade por meio de infraestrutura celular, avaliou-se como se comportava a cidade com as medidas que impunham restrição ou flexibilização da mobilidade, criando assim uma base de conhecimento com os melhores resultados para regular a mobilidade [7];

- O monitoramento do fluxo de transporte rodoviário, possibilitou avaliar o impacto da mobilidade na disseminação do vírus a partir da capital (epicentro), na fase de transmissão não comunitária, e sua relação com as rodovias interestaduais; além disso, utilizou este ambiente para avaliar os impactos econômicos baseados no padrão de mobilidade interurbana [4];

- A abordagem de monitoramento das notas fiscais eletrônicas permite avaliar o impacto econômico de políticas públicas, que interferiam na operação de setores econômicos. Por exemplo, na 1a semana da quarentena, o monitoramento indicou uma redução de faturamento das empresas em mais de 
25\% em relação a 2019 (Fig. 5), o que promoveu um acompanhamento e iniciativas que culminaram, em junho de 2020, no Plano São Paulo, que criou estratégias de abertura consciente de setores econômicos com base em indicadores do SIMI. A retomada da economia já foi percebida no final do próprio mês. Essa abordagem permitiu respostas mais ágeis (até uma semana), algo que antes só era possível em 60 dias.

- Cada secretaria realizou diferentes análises com o SIMI. Por exemplo, a Secretaria de Desenvolvimento Econômico viabilizou incentivos a setores mais críticos e criou programas de educação e incentivo a emprego em áreas mais afetadas.

- Por fim, os dados estão disponibilizados nos sites SIMI Dados Abertos $^{2}$ e SIMI Isolamento ${ }^{3}$, os quais são base para diversas pesquisas na academia.

A sala de situação representou uma referência de como o trabalho integrado entre secretarias, iniciativa privada e instituições de ensino e pesquisa podem contribuir para a criação de uma pauta estratégica de geração de informação para o processo de tomada de decisão. Em momentos de crise, esta sala pode suportar de forma eficiente o governo, usufruindo das vantagens oferecidas pelas Tecnologias da Informação e Comunicação. Espera-se que este modelo possa ser replicado em outros cenários que envolvam o monitoramento de cidades. $\mathrm{O}$ grupo de parceiros envolvidos neste ecossistema são:

- Mobilidade de Pessoas: Vivo; Claro; Tim; OI e ABR Telecom;

- Mobilidade veicular: Sec. de Logística e Transportes; ARTESP; DER; DERSA e WriteSYS;

- Saúde: Sec. de Saúde; SEADE; Microsoft e BCG consulting;

- Infraestrutura e gestão: Sec. de Desenvolvimento Econômico; IPT; Secretaria de Governo; PRODESP; USP; FAPESP e Tableau.

\subsection{Impacto social e relevância}

Toda a população paulista é impactada indiretamente por informações geradas pelo SIMI, as quais norteiam as decisões que afetam a operação de setores econômicos. A imprensa recorrentemente transmite estas informações para sociedade em telejornais ou outras mídias, como forma de disseminação de informação, inclusive ajudando na avaliação dos resultados e impactos gerados. Todo este processo de emprego de ciência e tecnologia demonstra rapidamente se as políticas públicas estão surtindo o efeito desejado, e provê uma rápida resposta aos governantes, a fim de permitir que tomem decisões mais assertivas.

Em termos de retorno de investimento social, onde se analisa os benefícios frente aos investimentos, podemos considerar que os benefícios gerados pelo SIMI são intangíveis, ou de difícil mensuração, pois fazem parte de uma estratégia estadual que objetiva reduzir os impactos da pandemia tanto na perspectivas da economia quanto na preservação de vidas. De acordo com [3], existem indícios que cada redução percentual nos índices de isolamento social refletem em vidas salvas, após 15 dias. Ainda de acordo com [4], o Plano São Paulo, ao estimular a retomada econômica, ajudou a preservar cerca de 318 mil postos de trabalho. Portanto, uma boa gestão baseada nos indicadores do SIMI pode produzir bons resultados nestas duas perspectivas.

\footnotetext{
${ }^{2}$ www.saopaulo.sp.gov.br/planosp/simi/dados-abertos/

${ }^{3}$ www.saopaulo.sp.gov.br/coronavirus/isolamento/
}

Em termos de investimento no SIMI, boa parte da sua infraestrutura era pré-existente no governo, ou foi obtida por meio de doações da iniciativa privada, ou mesmo de mecanismos oferecidos por projetos de pesquisas financiados pela FAPESP. No entanto, houve um grande envolvimento de capital humano de diversas entidades; por exemplo, os pesquisadores, alunos e professores do IPT dedicaram mais de 4 mil horas na sua criação e operação.

A ferramenta está em uso desde março de 2020 e perdurará por um período pós-pandemia para verificar o potencial de recuperação da economia, assim como as questões epidemiológicas (Fig. 6).

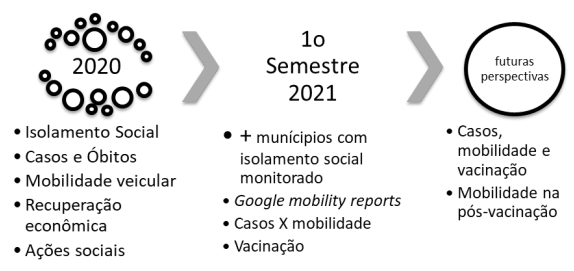

Figure 6: Cronograma do SIMI

\section{CONCLUSÕES}

No SIMI, as equipes multidisciplinares e a integração de dados foram essenciais para agregar e subsidiar os tomadores de decisão no enfrentamento da pandemia. O SIMI representou uma nova forma de abordar situações críticas baseada em ciência e tecnologia, utilizando forte integração entre diferentes entidades do governo e da iniciativa privada, com um objetivo comum: enfrentar a maior crise dos últimos tempos.

\section{REFERENCES}

[1] Arthur de M Del Esposte and et al. 2019. Design and evaluation of a scalable smart city software platform with large-scale simulations. Future Generation Computer Systems 93 (2019), 427-441. https://doi.org/10.1016/j.future.2018.10.026

[2] Rajvikram Madurai Elavarasan and Rishi Pugazhendhi. 2020. Restructured society and environment: A review on potential technological strategies to control the COVID-19 pandemic. Science of The Total Environment 725 (2020).

[3] Alexandre Gori Maia, Leticia Marteleto, Cristina Guimarães Rodrigues, and Luiz Gustavo Sereno. 2021. The short-term impacts of coronavirus quarantine in São Paulo: The health-economy trade-offs. PLOS ONE 16, 2 (02 2021), 1-18. https://doi.org/10.1371/journal.pone.0245011

[4] Eduardo Haddad, Renato Vieira, Inácio Araújo, Silvio Ichihara, Fernando Perobelli, and Karina Bugarin. [n.d.]. Economic Impacts of the São Paulo Plan (Technical Note). TD NEREUS 7-2020. Center for Regional and Urban Economics at the University of São Paulo (NEREUS).

[5] Moritz U. G. Kraemer, Chia-Hung Yang, Bernardo Gutierrez, and et al. 2020. The effect of human mobility and control measures on the COVID-19 epidemic in China. Science 368, 6490 (2020), 493-497. https://doi.org/10.1126/science.abb4218

[6] Nuria Oliver, Bruno Lepri, Harald Sterly, and et al. 2020. Mobile phone data for informing public health actions across the COVID-19 pandemic life cycle. Science Advances 6, 23 (2020).

[7] Gabriela Capobianco Palhares, Alessandro Santiago Santos, Eduardo Altomare Ariente, and Jefferson de Oliveira Gomes. [n.d.]. A privacy in times of pandemic and escalation of monitoring and tracing. Advanced Studies 34 ([n. d.]), 175-190. https://doi.org/10.1590/s0103-4014.2020.3499.011 (In Portuguese).

[8] Alessandro Santos, Igor Teixeira, Rodrigo Neves, Icaro Gonçales, Angelina Inacio, Eduardo Ueda, Eduardo Felipe Zambom Santana, Higor Souza, and Fabio Kon. 2021. Challenges and Strategies for Information Systems in the Decision-Making Process to Face the COVID-19 Pandemic: The São Paulo Case. 630-640. https: //doi.org/10.1007/978-3-030-72660-7_60

[9] M. Sulyok and M. Walker. 2020. Community movement and COVID-19: a global study using Google's Community Mobility Reports. Epidemiology and Infection 148 (2020), e284. https://doi.org/10.1017/S0950268820002757 